\title{
Probabilistic and Empirical Grounded Modeling of Agents in (Partial) Cooperative Traffic Scenarios
}

\author{
Claus Möbus, Mark Eilers ${ }^{1}$, Hilke Garbe, and Malte Zilinski ${ }^{2}$ \\ University of Oldenburg / OFFIS, Germany \\ \{FirstName.LastName\}@uni-oldenburg.de
}

\begin{abstract}
The Human Centered Design (HCD) of Partial Autonomous Driver Assistance Systems (PADAS) requires Digital Human Models (DHMs) of human control strategies for simulations of traffic scenarios. The scenarios can be regarded as problem situations with one or more (partial) cooperative problem solvers. According to their roles models can be descriptive or normative. We present new model architectures and applications and discuss the suitability of dynamic Bayesian networks as control models of traffic agents: Bayesian Autonomous Driver (BAD) models. Descriptive BAD models can be used for simulating human agents in conventional traffic scenarios with BetweenVehicle-Cooperation (BVC) and in new scenarios with In-Vehicle-Cooperation (IVC). Normative BAD models representing error free behavior of ideal human drivers (e.g. driving instructors) may be used in these new IVC scenarios as a first Bayesian approximation or prototype of a PADAS.
\end{abstract}

Keywords: digital human response models, probabilistic driver models, Bayesian autonomous driver models, learning of human control strategies, graphical modeling, human behavior learning and transfer, distributed cognition, mixtureof-experts model, visual attention allocation, partial cooperative problem solvers, partial autonomous assistance system, Bayesian assistance system, shared space, probabilistic detection of anomalies, driver assistance systems, traffic agents, dynamic Bayesian networks, hidden Markov models, betweenvehicle-cooperation, within-vehicle-cooperation.

\section{Introduction}

We discuss the suitability of a new type of real-time probabilistic control models for the psychological valid representation of traffic agent (e.g.: driver) behavior: Bayesian Autonomous Driver (BAD) models.

These models $[1,2]$ are developed in the tradition of Bayesian expert systems and Bayesian robot programming [3, 4]. Descriptive BAD models can be used for simulating agents in conventional traffic scenarios with Between-Vehicle- Cooperation

\footnotetext{
${ }^{1}$ Project Integrated Modeling for Safe Transportation (IMOST) sponsored by the Government of Lower Saxony, Germany under contracts ZN2245, ZN2253, ZN2366.

${ }^{2}$ Project ISi-PADAS funded by the European Commission in the 7th Framework Program, Theme 7 Transport FP7-218552.
} 
(BVC). Furthermore, when modeling normative correct behavior of ideal human drivers (e.g. driving instructors) they may be used for a conceptual new kind of systems: Bayesian Assistance Systems (BAS). These may be used for In-VehicleCooperation (IVC) between the human driver and the BAS. Thus a BAS may be regarded as a first Bayesian approximation or prototype of a PADAS. Due to their probabilistic nature BAS can not only be used for real-time control but also for realtime detection of anomalies in driver behavior and real-time generation of supportive interventions.

Traffic scenarios can be regarded as problem situations with one or more (partial) cooperative problem solvers. A scenario is called cooperative, when all problemsolving agents try to solve a goal specified by one single principal. Thus, a scenario is partial cooperative, when goals are defined by several different principals [5]. Successful problem solutions require (nonverbal) communication and distributed cognition. This is especially true when traffic scenarios are deregulated as in the IVC (driver - PADAS - interaction) or in the BVC (e.g. shared space) type.

In most cases, traffic maneuvers are run without risk. Though, risky situations can occur anytime. We call risky maneuvers anomalies which only experienced drivers are able to prevent or to anticipate automatically. Other drivers probably cannot and therefore might need support generated by a PADAS. It is expected that assistance systems will enhance situation awareness, cooperation and driving competence of unskilled or non-cooperative drivers in the near future. Thus the design challenge of intelligent assistance systems should aim at modeling traffic agents with their beliefs, expectations, behavior, situation awareness, and their skills to recognize situations, to diagnose and prevent anomalies. We think that dynamic probabilistic models are appropriate for these challenges. We review some types of models and propose a new mixture-of-expert architecture with attention allocation stemming from our current research $[1,2]$.

\section{Distributed Cognition and Traffic Scenarios}

The concept of distributed cognition was introduced in the mid 1980s by Edwin Hutchins [6]. His theory proposes that human knowledge and cognition are not confined to the individual. Instead, they are distributed by placing cognitive skills on the objects, individuals, and tools in the environment. Cognitive processes may be distributed across the members of a social group or may be distributed in the sense that the operation of the cognitive system involves coordination between internal and external (material or environmental) structure.

\subsection{Cooperative Scenarios: Crews and In-Vehicle-Dyads}

Hutchins [6] studied crews with an emphasis on anthropological and nonexperimental methods. These methods then became popular. The question raised was how crews of ships can function as a distributed machine, offloading the cognitive burden of ship navigation onto each member of the crew. Hutchins approach questioned disembodied views of cognition and alternatively suggested studying cognitive systems that are composed of multiple agents and the material world. Later studies 
generalized the domains and put an emphasis on airline cockpits crews and humancomputer interaction scenarios.

Members of a public traffic scenario with BVC do not form a stable social group but rather an ad hoc group with a limited life time and a limited communication vocabulary. Whereas members in a nonpublic traffic scenario (novice driver and driving instructor; Fig. 1) with IVC form a stable social group which resembles a crew.

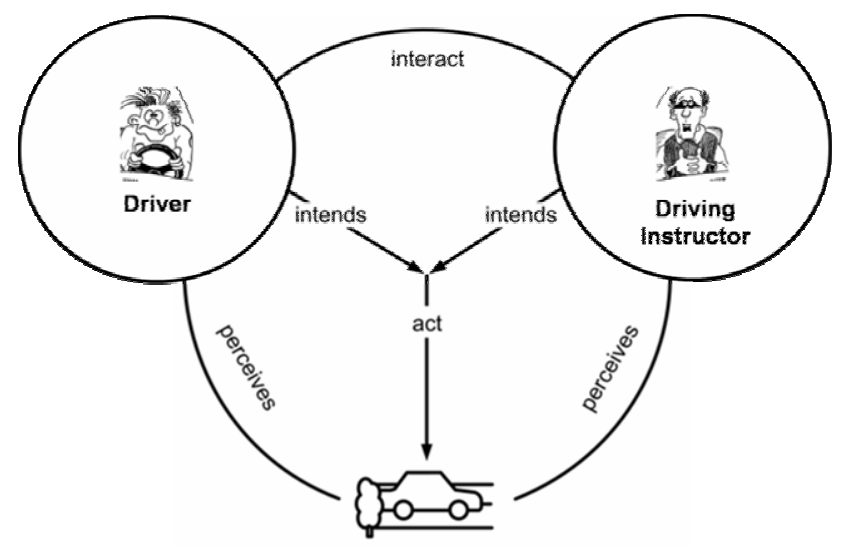

Fig. 1. Cooperative driving scenario with in-vehicle-cooperation (background graphics from [7] with kind permission of the publisher)

\subsection{Partial Cooperative Scenarios: Ad-Hoc Groups and Shared Space}

Crews on navigation bridges or in aircraft cockpits work in agreement with a single principal. They form a cohesive group whose members normally cooperate for hours in solving the problems arising during ship or aircraft operation. This cooperation includes exchange of complex verbal messages which require a high dimensional state space for the agent models.

Public traffic scenarios are of a fundamentally different kind. Communication, cooperation and the action repertoire is limited in amount and complexity. Agents are their own principals and do not belong to a formal cohesive group. They come together by chance and (might) try to maximize their personal utilities; sometimes ignoring the needs of others. Internal group norms are substituted by traffic rules which are expected to accelerate negotiations between the traffic agents in a scenario. The solution to a traffic coordination problem is a distributed but synchronized sequence of sets of actions (e.g. collision-free crossing an intersection) emitted by autonomous agents.

Shared space describes an approach to the design, management and maintenance of public spaces which reduces the adverse effects of conventional traffic engineering by stimulating the situation awareness of all traffic agents. The shared space approach is based on the observation that individuals' behavior in traffic is more positively affected by the built environment of the public space than by conventional traffic control devices (signals, signs, road markings, etc.) or regulations. An explanation for the apparent paradox that a reduction in regulation leads to safer roads may be found by studying the risk compensation effect: "Shared Space is successful because the 
perception of risk may be a means or even a prerequisite for increasing public traffic safety. Because when a situation feels unsafe, people are more alert and there are fewer accidents." (http://www.shared-space.org/; visited 23.02.2009).

\section{Modeling Agents in (Partial) Cooperative Scenarios}

Skilled agents differ from novices in their competence of risk perception thus increasing their personal safety. Computational agent models have to represent these and other kinds of perceptions, beliefs, goals and actions of the ego agent and alter agents. Driver models should

- predict and generate driver behavior emitted by individual drivers sometimes in interaction with assistance systems

- identify situations or maneuvers and classify behavior (e.g. anomalous vs. normal) of ego driver

- provide a robust and valid mapping from human sensory data to human control actions

- be learnt from time series of raw data or empirical probability distributions with statistical sound (machine-learning) procedures with only a few non-testable ad hoc or axiomatic assumptions

- should be able to learn new patterns of behavior without forgetting already learnt skills (stability-plasticity dilemma).

A driver is a human agent whose skills can be described by the cognitive, associative, and autonomous stage. Accordingly various modeling approaches are adequate: production-system (e.g. models in a rule-based architecture [8 - 10]), control-theoretic $[11,12]$, and probabilistic models [13, 14]. The advantage of probabilistic models is that they fulfill the above criteria especially that they are more robust than other approaches. This is a great advantage due to the irreducible incompleteness of knowledge about the environment and the underlying psychological mechanisms [4].

\subsection{Bayesian Assistance Models in In-Vehicle-Dyads}

As an example we present a result of Rizzo et al. [15]. The authors studied the behavior of drivers suffering from Alzheimer disease. At a lane crossing a car incurred from the right (Fig. 2). Many maneuvers of the Alzheimer patients ended in a collision, as they suffered from the looking without seeing syndrome. The modeling task should lead to a probabilistic BAS model, which is diagnosing and correcting the anomalous behavior of the inexperienced driver. Fig. 2 demonstrates the behavioral risk assessment in probabilistic terms and Fig. 3 the replacement of the real driving inspector by the corresponding BAS model an intended Bayesian prototype of a PADAS.

How can this model be derived by methods of Bayesian driver modeling and what is the use of it? The best way to explain this is an obstacle scenario which is known to generate intention conflicts within the driver (Fig. 4). When an obstacle (animal, car) is appearing unexpectedly people autonomously react with a maneuver M-- which is not recommended by experts. M-- drivers try to avoid collisions even at high 


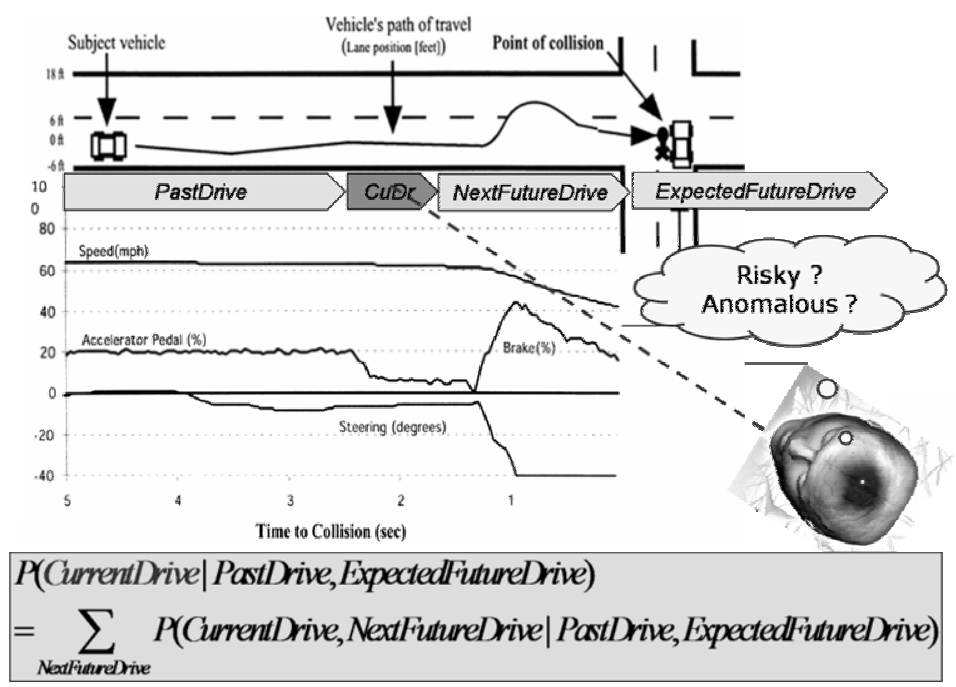

Fig. 2. Driving behavior of an Alzheimer driver in a simulated intersection incursion [15] and risk assessment by a probabilistic normative Bayesian driver model residing in the subject vehicle

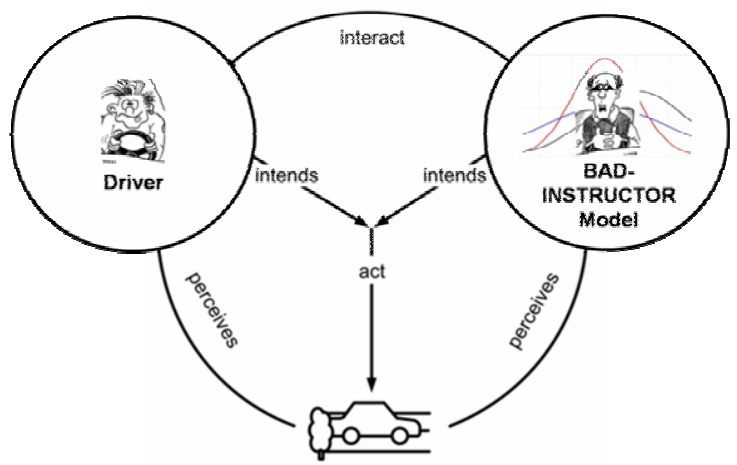

Fig. 3. Cooperative driving scenario with in-vehicle-cooperation between non-expert driver and Bayesian normative driver model (BAS prototype) (background graphics from [7] with kind permission of the publisher)

velocities by steering to the left or right risking a fatal turnover. The recommended maneuver $\mathrm{M}+$ includes the hold and brake sub-maneuvers. When drivers are instructed to drive $\mathrm{M}+$ these data provide the learning data for the BAS version of the PADAS according the methods of 3.2.

With an existing BAS model a worst-case scenario is planned to test the services of the BAS. Drivers are instructed not to drive according to $\mathrm{M}+($ not $\mathrm{M}+=\mathrm{M}--)$. Because of the probabilistic nature of the BAS it is possible to compute the conditional probability $\mathrm{P}\left(\right.$ Action $\left._{\mathrm{t}} \mid \mathrm{M}+\right)$. This is a measure of the anomaly of the driver behavior 


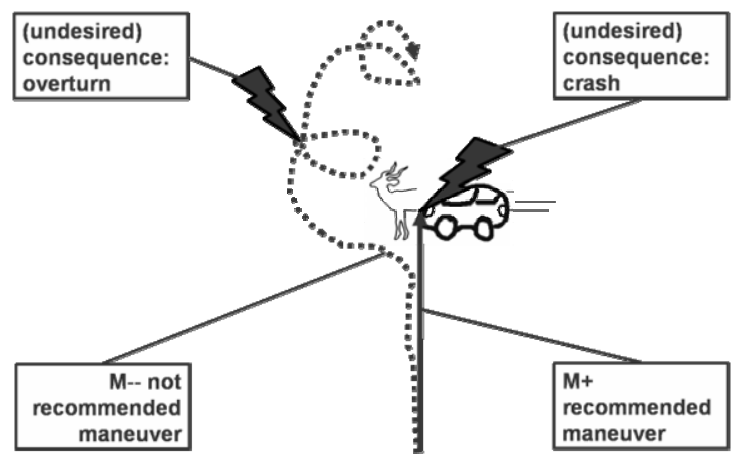

Fig. 4. Scenario with conflicting maneuvers

under the hypothesis that the observed actions are generated by following the correct maneuver M+.

\subsection{Bayesian Autonomous Driver (BAD) Models}

Due to the variability of human cognition and behavior and the present time irreducible lack of knowledge about cognitive mechanisms it seems rational to conceptualizes, estimate and implement probabilistic models when modeling traffic agents. In contrast to other models probabilistic models could be derived objectively from the empirical distributions of the random variables of interest with only a few axiomatic assumptions. Model validity is thus included in the modeling process by model-driven data-analysis without any ex-post validation. BAD models describe phenomena on the basis of variables and conditional probability distributions (JPDs). This is in contrast to models in cognitive architectures (e.g. ACT-R) which try to simulate cognitive algorithms and processes on a granular basis which are difficult to identify with e.g. functional magnetic resonance imaging (FMRI) methods [16, 17]. Instead a more abstract mapping is possible: the mapping of the activation of entire ACT-R-modules into the states of a Hidden Markov Model (Fig. 5). At present these activations are the only dynamic aspects of ACT-R-models which could be empirically identified by brain imaging techniques $[16,17]$.

\subsubsection{Hidden Markov Models (HMMs)}

Currently we are evaluating the suitability of static and dynamic graphical models known as Hidden Markov Models (HMMs) or Bayesian Belief Nets (BBNs). With the static type it is possible to generate reactive models [3] and inverse (naïve) [18] models. Currently our research [1,2] has shown that static models generate behavior which is too erratic for human behavior. As a consequence we focus ourselves on the dynamic type of real-time control for simulated cars [2].

The dynamic type enables the creation of Markov Models (MMs), Hidden Markov Models (HMMs) [19-21], Input-Output-HMMs (IOHMMs) [22], Reactive IOHMMs (RIOHMMs; Fig. 5), Coupled RIOHMMs (CRIOHMMs; Fig. 6), [23]. HMMs allow the recognition of situations, goals and intentions and the generation of behavior of Belief-Desire-Intention (BDI-) Agents. RIOHMMs implement driver models e.g. with 
ACT-R module activations. The two arrows into the random variable nodes $\mathrm{Z}_{\mathrm{j}}$ denote the combined dependence of actions on sensory inputs and activations of hidden ACT-R modules or brain regions. Even if module activations were known sensory inputs are still necessary to propose actions. CRIOHMMs model dyads of agents with mutual belief influences. The belief state of each agent depends only on his own history and on the belief state of his partner. Whether it is plausible has to be tested by conditional independence hypotheses. Within each agent the model is of the RIOHMM-type.

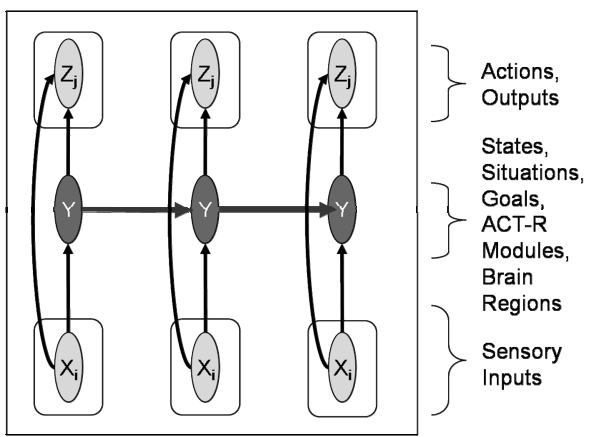

Fig. 5. Reactive Input-Output HMM (RIOHMM) slightly simplified version of Bengio and Frasconi [22]

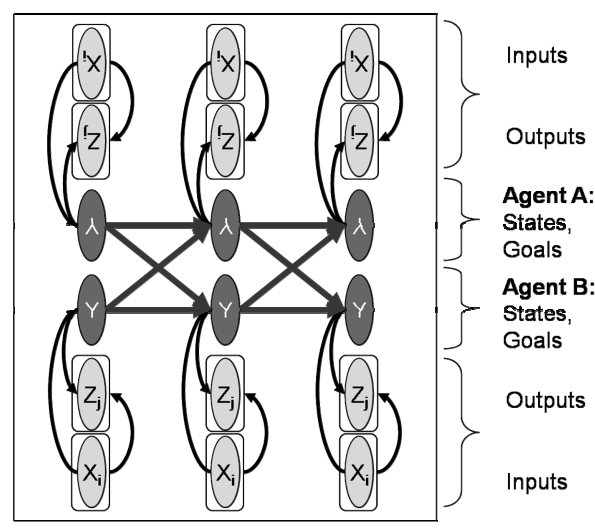

Fig. 6. Coupled Reactive Input-Output Hidden Markov Models (CRIOHMM)

There is a trade-off between HMMs and DBNs. Inferences in HMMs are more efficient than in DBNs, whereas the state-space in HMMs grows more rapidly than in corresponding DBNs. This is especially true, when the HMM is used not only for situation recognition but also for real-time control of behavior.

\subsubsection{Dynamic Bayes Net Models (DBNs)}

In our current research [2] we strive for the realization of the dynamic Bayesian model (Fig. 7). It implements the sensory-motor system of human drivers in the functional autonomous layer or stage of Anderson [24]. It is a psychological motivated mixture-of-experts (= mixture-of-schema) model with autonomous and goal-based attention allocation processes. It implements the autonomous layer of a cognitive agent, is distributed across two time slices, and avoids the latent state assumptions of HMMs. Learning data are time series of relevant variables: percepts, goals, and actions. We can model individual or groups of human and artificial agents.

The model propagates information in various directions. When working top-down, goals emitted by the associative layer select a corresponding expert (schema), which propagates actions, relevance of areas of interest (AoIs) and perceptions. When working bottom-up, percepts trigger AoIs, actions, experts and goals. When the task or goal is defined and the model has certain percepts evidence can be propagated simultaneously top-down and bottom-up and the appropriate expert (schema) and its 
behavior can be activated. Thus, the model can be easily extended to implement a modified version of the SEEV visual scanning or attention allocation model of Horrey, Wickens, and Consalus [25]. Please note that due to our modification the indices have changed. In contrast to Horrey et al. the model can predict the probability of attending a certain AoI on the basis of single, mixed, and even incomplete evidence (goal priorities, percepts, effort to switch between AoIs).

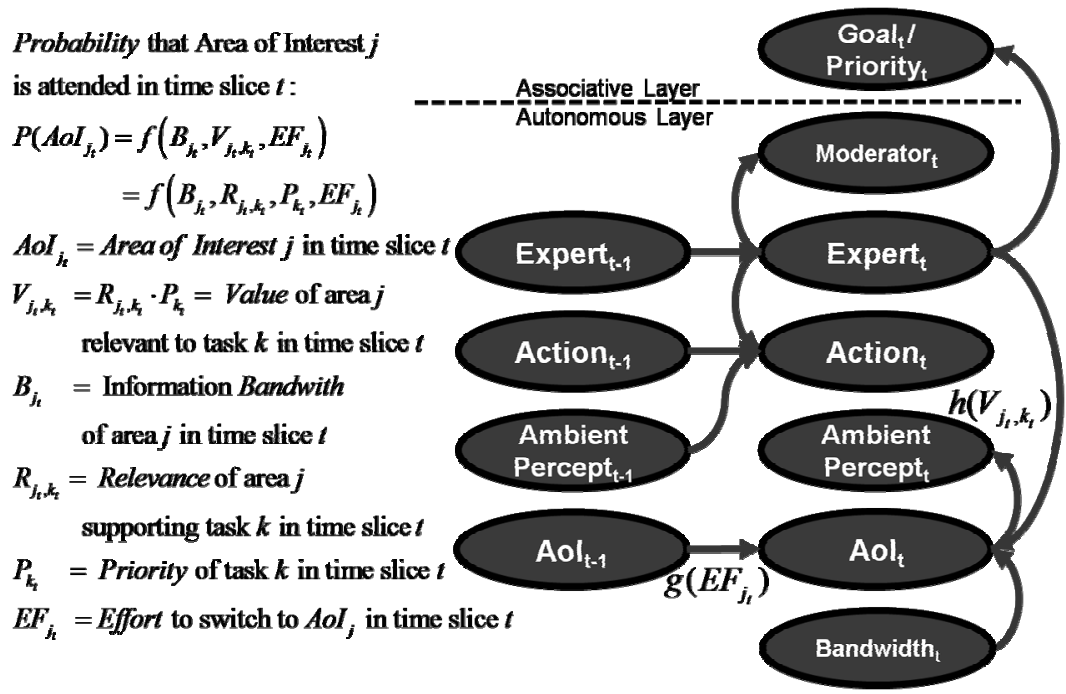

Fig. 7. Mixture-of-Experts (= Mixture-of-Schema) Architecture of Bayesian Autonomous Driver (BAD) Model with visual attention allocation extension (mapping ideas of Horrey et al. [25] into the Bayesian network domain)

There are various scientific challenges designing and implementing BAD Models. The first main challenge is to generate driver behavior by a mixture-of-expert architecture. While mixture-of-experts approaches are known from pattern classification [26] it is the first time that this approach is used in human modeling. The second main challenge is that we want to integrate from psychological action control theory various perceptional invariants known as tau-measures [27] into a computational human model. In conventional models variables with different dimensions (distances, angles, times, changes, etc) are input to the models. Tau measures transform all non-time measures into the time domain. Some measures are already used in engineering (TTC, TTLC).

The role of these invariants for the psychology of motion control is discussed since Lee [28]. Now it is the first time that these measures are used to generate behavior in a probabilistic mixture-of-expert (MoE) model. In a MoE model it is assumed that the behavior can be context dependent generated as a mixture of ideal schematic behaviors (= experts). Thus the stability/plasticity dilemma [29] of neural network models is avoided. New behavior can be learnt by adding a new expert to the library of expert. Experts do not influence each other directly. Pure expert behavior without 
any additional mixture component is shown only in typical pure situations (e.g. the perception of a hair pin triggers the hair pin model expert).

All probabilistic models presented here can be constructed by data mining single or aggregated driver's behavior traces in experimental settings with or without experimental induced goals.

\section{Summary}

We discussed two kinds of (partial) cooperative traffic scenarios with within-vehicle(driving school) and between-vehicle-cooperation (Shared Space). Either individual or groups of human agents can be modeled by Bayesian Autonomous Agent (BAD) models according to the Bayesian Programming Approach. Learning data are time series of pertinent variables: percepts, goals, and actions. Modeling ideal correct behavior may provide the basis for Bayesian prototypes for partial autonomous assistance systems (BAS Models). Because of the probabilistic nature of the BAS it is possible to compute the conditional probability $\mathrm{P}\left(\right.$ Action $\left._{t} \mid \mathrm{M}+\right)$ of the anomaly of the driver behavior under the hypothesis that the observed actions are generated by interpreting the correct maneuver $\mathrm{M}+$. This makes it possible to define thresholds for PADAS interventions.

\section{References}

1. Möbus, C., Eilers, M.: First Steps Towards Driver Modeling According to the Bayesian Programming Approach. In: Urbas, L., Goschke, T., Velichkovsky, B. (eds.) KogWis 2008, Tagungsband der 9. Fachtagung der Gesellschaft für Kognitionswissenschaft, p. 59. Verlag Hille, Dresden (2008)

2. Möbus, C., Eilers, M.: Further Steps Towards Driver Modeling according to the Bayesian Programming Approach. In: Conference Proceedings, HCI 2009, Digital Human Modeling. LNCS (LNAI). Springer, San Diego (2009) (accepted)

3. Lebeltel, B., Mazer, D.: Bayesian Robot Programming. Autonomous Robots 16, 49-79 (2004)

4. Bessiere, P., Laugier, C., Siegwart, R. (eds.): Probabilistic Reasoning and Decision Making in Sensory-Motor Systems. Springer, Berlin (2008)

5. Xiang, Y.: Probabilistic Reasoning in Multiagent Systems - A Graphical Models Approach. Cambridge University Press, Cambridge (2002)

6. Hutchins, E.: Cognition in the Wild. MIT Press, Cambridge (1995)

7. Löper, C., Kelsch, J., Flemisch, F.O.: Kooperative, Manöverbasierte Automation und Arbitrierung als Bausteine für hochautomatisiertes Fahren, In: Gesamtzentrum für Verkehr Braunschweig (Hrsgb): Automatisierungs-, Assistenzsysteme und eingebettete Systeme für Transportmittel, GZVB, Braunschweig S, pp. 215-237 (2008)

8. Gluck, K.A., Pew, R.W.: Modeling Human Behavior with Integrated Cognitive Architectures. Lawrence Erlbaum Associates, Mahwah (2005)

9. Anderson, J.R.: How Can the Human Mind Occur in the Physical Universe? Oxford University Press, Oxford (2007) 
10. Baumann, M., Colonius, H., Hungar, H., Köster, F., Langner, M., Lüdtke, A., Möbus, C., Peinke, J., Puch, S., Schießl, C., Steenken, R., Weber, L.: Integrated Modeling for Safe Transportation - Driver modeling and driver experiments (in press). Fortschrittsbericht des VDI in der Reihe 22 (Mensch-Maschine-Systeme)

11. Bischof, N.: Struktur und Bedeutung: Einführung. Huber, Systemtheorie (1995)

12. Jagacinski, R.J., Flach, J.M.: Control Theory for Humans: Quantitative Approaches to Modeling performance. Lawrence Erlbaum Associates, Mahwah (2003)

13. Wickens, T.D.: Models for Behavior: Stochastic Processes in Psychology. Freeman, San Francisco (1982)

14. Gopnik, A., Tenenbaum, J.B.: Bayesian networks, Bayesian learning and cognitive development. Development Science 10(3), 281-287 (2007)

15. Rizzo, M., McGehee, D.V., Dawson, J.D., Anderson, S.N.: Simulated Car Crashes at Intersections in Drivers With Alzheimer Disease. Alzheimer Disease and Associated Disorders 15(1), 10-20 (2001)

16. Anderson, J.R., Fincham, J.M., Qin, Y., Stocco, A.: A Central circuit of the mind. Trends in Cognitive Science 12(4), 136-143 (2008)

17. Quin, Y., Bothell, D., Anderson, J.R.: ACT-R meets fMRI. In: Zhong, N., Liu, J., Yao, Y., Wu, J., Lu, S., Li, K. (eds.) Web Intelligence Meets Brain Informatics. LNCS (LNAI), vol. 4845, pp. 205-222. Springer, Heidelberg (2007)

18. Le Hy, R., Arrigoni, A., Bessière, P., Lebeltel, O.: Teaching Bayesian Behaviours to Video Game Characters. In: Robotics and Autonom. Systems, vol. 47, pp. 177-185. Elsevier, Amsterdam (2004)

19. Oliver, N., Pentland, A.P.: Graphical Models for Driver Behavior Recognition in a SmartCar. In: IEEE Intl. Conf. Intelligent Vehicles, pp. 7-12 (2000)

20. Miyazaki, T., Kodama, T., Furushahi, T., Ohno, H.: Modeling of Human Behaviors in Real Driving Situations. In: 2001 IEEE Intelligent Transportation Systems Conference Proceedings, pp. 643-645 (2001)

21. Kumugai, T., Sakaguchi, Y., Okuwa, M., Akamatsu, M.: Prediction of Driving Behavior through Probabilistic Inference. In: Proceedings of the $8^{\text {th }}$ International Conference on Engineering Applications of Neural Networks (EANN 2003), pp. 117-123 (2003)

22. Bengio, Y., Frasconi, P.: Input/output Hidden Markov Models for Sequence Processing. IEEE Transactions on Neural Networks 7, 1231-1249 (1996)

23. Oliver, N.M.: Towards Perceptual Intelligence: Statistical Modeling of Human Individual and Interactive Behaviors, MIT Ph.D. Thesis (2000)

24. Anderson, J.R.: Learning and Memory. John Wiley, Chichester (2002)

25. Horrey, W.J., Wickens, C.D., Consalus, K.P.: Modeling Driver's Visual Attention Allocation While Interacting With In-Vehicle Technologies. J. Exp. Psych. 12, 67-78 (2006)

26. Bishop, C.M.: Pattern Recognition and machine learning. Springer, Heidelberg (2006)

27. Lee, D.N.: How movement is guided (2006), http: / /www.perception-in-action.ed.ac.uk/publications.htm

28. Lee, D.N.: A theory of visual control of braking based on information about time-tocollision. Perception 5, 437-459 (1976)

29. Hamker, F.H.: RBF learning in a non-stationary environment: the stability-plasticity dilemma. In: Howlett, R.J., Jain, L.C. (eds.) Radial Basis Function networks 1: Recent Developments in Theory and Applications; Studies in fuzziness and soft computing, ch. 9, vol. 66, pp. 219-251. Physica Verlag, Heidelberg (2001) 